\title{
Littérature et médecine. Le cas de Proust, dir.
} M. NATUREL

\section{Eleonora Di Mauro}

\section{OpenEdition}

\section{Journals}

\section{Edizione digitale}

URL: https://journals.openedition.org/studifrancesi/23012

DOI: 10.4000/studifrancesi.23012

ISSN: 2421-5856

\section{Editore}

Rosenberg \& Sellier

\section{Edizione cartacea}

Data di pubblicazione: 1 avril 2020

Paginazione: $210-211$

ISSN: 0039-2944

\section{Notizia bibliografica digitale}

Eleonora Di Mauro, «Littérature et médecine. Le cas de Proust, dir. M. natureL», Studi Francesi [Online], 190 (LXIV | I) | 2020, online dal 01 mai 2020, consultato il 02 août 2021. URL: http://

journals.openedition.org/studifrancesi/23012 ; DOI: https://doi.org/10.4000/studifrancesi.23012

Questo documento è stato generato automaticamente il 2 août 2021.

\section{(9) $\odot \Theta \Theta$}

Studi Francesi è distribuita con Licenza Creative Commons Attribuzione - Non commerciale - Non opere derivate 4.0 Internazionale. 


\title{
Littérature et médecine. Le cas de Proust, dir. M. NATUREL
}

\author{
Eleonora Di Mauro
}

\section{NOTIZIA}

Littérature et médecine. Le cas de Proust, dir. M. NATUREL, Paris, Hermann, 2018, 344 pp.

1 I contributi di questa miscellanea hanno l'obiettivo di mettere in luce le numerose interconnessioni tra il Proust autore e l'uomo, la sua infanzia segnata dall'autorità del padre medico, l'asma come malattia debilitante psicosomatica e, più in generale, $\mathrm{i}$ rapporti tra nevrastenia e creazione letteraria, tra intermittenze del cuore e cardiologia.

2 Fin dalla prefazione Littérature et savoirs ou le savoir de la littérature (pp. 9-23), Margery VIBE SKAGEN indaga i rapporti tra letteratura e scienza, ripercorrendo diacronicamente gli antagonismi e le affinità che hanno storicamente caratterizzato il rapporto tra le due discipline. L'Avant-propos di Mireille NATUREL (pp. 25-28) chiarisce innanzitutto le ragioni alla base della profonda influenza che la medicina in quanto scienza ha esercitato su Marcel Proust: figlio e fratello di medici infatti, si rileva presso l'autore un'osservazione clinica dei moti del cuore, con un approccio analogo alla precisione chirurgica. Nell'introduzione (La guérison par la lecture, pp. 29-39) invece, Michel SCHNEIDER riflette sul carattere terapeutico intrinseco alla lettura della Recherche, identificando Proust quale "medico dell'anima", professore di memoria e di desiderio.

La miscellanea si articola in seguito in cinque sezioni rispettivamente intitolate: «L'écriture et la maladie», «Des influences médicales et littéraires», «Proust médecin», «États du corps, états psychiques» e infine «Pathologies réelles et pathologies imaginaires».

4 La prima sezione si apre con un contributo di Edward BIZUB (La psychothérapie de Proust: une œuvre d'art?, pp. 43-57) che mette in luce la concezione proustiana del romanzo, 
inteso come strumento terapeutico attraverso cui l'autore sarebbe stato in grado di esplorare il proprio inconscio. Il racconto assume dunque i contorni di un viaggio alla scoperta dell'Io, anche tramite l'osservazione di sintomi di natura fisiologica, dove il corpo emerge quale veicolo espressivo della memoria involontaria.

5 L'intervento di Philippe CHARDIN (Le névropathe et son Esculape: un duo romanesque des années 1920, pp. 59-79) evidenzia invece la diffusione, in epoca moderna, di un genere letterario che non disdegna un interesse per la medicina, come dimostrano alcuni romanzi coevi tra cui La montagna incantata di Mann o La coscienza di Zeno di Svevo. Tuttavia, lo studioso precisa l'atteggiamento ambivalente di Proust nei confronti della medicina: da un lato è evidente il fascino esercitato dalla scienza sullo scrittore; d'altro canto però, perplesso di fronte all'insorgenza di un male ai tempi non catalogabile e ritenuto psicosomatico quale l'asma, Proust riflette sui limiti della disciplina medica e si interroga piuttosto sul carattere creativo della sofferenza, intesa come veicolo indispensabile a qualsivoglia forza intellettuale.

6 La seconda sezione, "Des influences médicales et littéraires», si apre poi con un contributo di Luc FRAISSE (Proust et les pères médecins: le poids d'un jugement, pp. 83-104) che indaga l'influenza delle opere scientifiche di Proust padre, stabilendo un parallelo tra il carattere psicosomatico dell'asma, intesa come responsabilità del paziente proprio in virtù del suo carattere psicogeno e in seguito come vero e proprio veicolo di conoscenza dell'enigma dell'Io. Nell'intervento successivo invece (Le docteur Doyen, pp. 105-108), Stéphane BALCEROWIAK esplora la possibile influenza dell'eccentrico medico parigino sull'opera proustiana.

7 La terza sezione, dedicata, come anticipato, al Proust "medico", si schiude con un'analisi di Jean-Pierre OLlivier (Les mots du cœur, pp. 131-156) che cataloga le ricorrenze di alcuni termini chiave all'interno della Recherche: uno su tutti, il sostantivo cœur che ricorre in più di cinquecento occasioni ed è investito del ruolo di fornire le prove della dislocazione corporale di emozioni inizialmente psicologiche. Successivamente Béatrice ATHIAS (La voix comme "pharmakon", pp. 157-175) esplora il carattere ambivalente affidato alla voce umana: quando femminile, essa è ricercata come oggetto vitale in ricordo della voce materna, ma talvolta viene esperita anche come spaventoso veicolo di violenza verbale rispetto al quale il soggetto è inerme e indifeso.

8 In Neurasthénie et mélancolie dans la pensée clinique du XIX siècle (pp. 177-195), Julie MUELLER analizza le differenze che intercorrono tra due istanze costantemente trattate nell'opera proustiana: la nevrastenia e la malinconia, dove quest'ultima è considerata il vero motore delle intermittenze del cuore nella sua accezione aristotelica di phantasia, ovvero un movimento dell'animo prodotto da una sensazione in atto. In Marcel Proust, romancier de l'hystérie (pp. 197-225), Samuel LAPASTIER indaga l'influenza degli studi sull'isteria di Charcot per la creazione del personaggio del Dottor Boulbon, dando prova della presenza di Proust padre alle lezioni di Charcot alla Salpêtrière.

9 La quarta sezione «États du corps, états psychiques» si apre con un contributo di Sief HOUPPERMANS (Désirs affolés, pp. 229-241) in cui lo studioso esplora la gelosia come elemento inestricabilmente legato alle funzioni vitali e, più precisamente, alla respirazione, mentre, in Tandis qu'ils agonisent: Proust et les malades imaginaires ou sérieux (pp. 243-261), Isabelle DUMAS rileva la centralità di sadismo e crudeltà quali istanze 
caratterizzanti il discorso del narratore sui malati rappresentati dalla zia Léonie, da Swann e dalla nonna del protagonista.

Nella quinta e ultima sezione, «Pathologies réelles et pathologies imaginaires», Gabrielle ROY-CHEVARIER (Voyage autour du lit du malade, pp. 265-277) riflette sulla concezione proustiana della malattia come stato di grazia e come strumento privilegiato atto ad avvicinare l'artista alla propria anima, mentre il contributo seguente, ad opera di Dagmar WIESER (De l'obésité et du stress: Proust, Ali-Bab et le docteur Babinski, pp. 279-312) indaga la relazione esistente tra obesità e stress, su cui l'autore della Recherche riflette in maniera oggettiva all'interno dell'opera, caratterizzando alcuni dei propri fenotipi anche grazie agli studi del neurologo Babinski. Infine, nella postfazione Littérature et savoirs: le cas du viellissement (pp. 313-321), Margery VIBE SKAGEN rileva l'utilità della letteratura come canale per trascendere le categorie dell'età, e dunque dell'invecchiamento e della malattia, al fine di costruire un sapere estetico, etico, esistenziale, individualizzato e storico del senso del tramonto. 